\title{
Smart streetlights in Smart City: a case study of Sheffield
}

\author{
Eisley Dizon ${ }^{1} \cdot$ Bernardi Pranggono $^{1}$ (i)
}

Received: 25 July 2020 / Accepted: 9 February 2021 / Published online: 24 February 2021

(c) The Author(s) 2021

\begin{abstract}
Smart streetlights can be used to enhance public safety and well-being. However, not only it is one of the most draining structures in terms of electricity, but it is also economically straining to local government. Typically, many councils adopt a static or conventional approach to street lighting, this presents many inefficiencies as it does not take into account environmental factors such as light levels and traffic flows. This paper will present the utilities of a streetlights in Sheffield and how different councils tackle the issue by using different lighting schemes. Investigation of current implementations of information and communication technologies (ICT) such as Internet of Things (IoT) in streetlights will be necessary to understand different proposed models that are used in 'smart' street lighting infrastructure. Case studies from Doncaster and Edinburgh are explored as they are using similar technology and having a similar sized topology as Sheffield. To analyze different models, StreetlightSim, an open-source streetlight simulator, is used to present different lighting schemes. There will be four time-based schemes: Conventional, Dynadimmer, Chronosense and Part-Night which have varying capabilities that will be simulated to present a plethora of solutions for Sheffield's street lighting problem. The results from the simulations showed mixed readings, the time-based schemes showed reliable data from StreetlightSim's own evaluations, however its adaptive approach will need to be further analyzed to demonstrate its full capability.
\end{abstract}

Keywords Internet of things $\cdot$ Streetlight $\cdot$ Smart city $\cdot$ Wireless sensor networks

\section{Introduction}

Population growth and urbanization have been drastically increasing over recent years, more people seeking better opportunities in cities. Currently $5 \%$ of the world population are residing in cities, increasing to two thirds by 2050 (UnitedNations 2019). That is roughly an additional 2.5 billion people living in cities. With the huge increase in people living in cities, comes with a cost and demand for the government. There will be a larger amount of energy needed to be provided to maintain public safety and services. Moreover, with fossil fuels becoming scarcer and the continuous inflation of its price, better power management and a strategy to become more energy efficient needs to be implemented.

To reduce energy comsumption, local government bodies are now replacing high pressure sodium (HPS) lamps with light emitting diode (LED) technologies with lower power

Bernardi Pranggono

b.pranggono@shu.ac.uk

1 Department of Engineering and Mathematics, Sheffield Hallam University, Sheffield, UK consumption in streetlights, improving lighting efficiency by a factor of five (Escolar et al. 2014). It is also indicated that the lifetime of LED lamps being over ten times to its HPS counterpart, in turn reducing maintenance (Masoud 2015). However, this is still not enough to meet cities energy reduction targets as streetlights can consume up to 43.9 billion $\mathrm{kWh}$ of electricity every year (Liu 2014) being a major contributor to energy consumption in cities, taking up to nearly $30 \%$ of energy consumption for any country (Badgelwar and Pande 2017). Currently Sheffield City Council have replaced most of their old HPS lamps with LED lamps, installing 66,802 units and encompassing $99.5 \%$ of their streetlights infrastructure (SheffieldCityCouncil 2020).

Internet of Things (IoT) has been implemented in various areas including in smart city (Talari et al. 2017; Zanella et al. 2014). Arguably, smart streetlights are the platform of smart city as it can have many features to support a smart city. Intelligently design streetlight not only can reduce electricity usage in the city but also enhance public safety and wellbeing. This paper focusing on the energy efficiency introduced in the smart city due to the implementation of 
smart streetlights using information and communication technologies (ICT) such as IoT.

The paper studies the smart streetlights implementation in Sheffield streetlight network. The level of energysavings is investigated and compared with different lighting schemes. There will be four time-based schemes: Conventional, Dynadimmer, Chronosense and Part-Night. An opensource simulator, the StreetlightSim (Lau et al. 2014) will be used to evaluate the different schemes. To the best of our knowledge, there is no similar work that used Sheffield as a case study.

The remainder of the paper is organized as follows. Section 2 presents background on IoT and smart streetlighting. Section 3 presents our research methodology. In Sect. 4, we study IoT-based streetlights implementation in smart city with Sheffield as a study case. Finally, conclusion of this paper is presented in Sect. 5

\section{Background}

\subsection{Internet of Things}

Since first coined by Kevin Ashton in 1999 (Ashton 2009; Boulaalam 2019), there are various definitions of the IoT exists. In essence, IoT can be define loosely as any devices or objects equipped with sensors which can exchange information through the network (Internet) without human intervention (Atzori et al. 2010; Feki et al. 2013; Gubbi et al. 2013; Javed et al. 2018). IoT communicate device to another device or connect machine-to-machine (M2M). Various IoT implementation is in place from smart home to smart agriculture, from eHealth to industry 4.0 (Borgia 2014).

IoT, also known as the Internet of Everything, is essentially a blanket term for all devices and network infrastructure, that are connected to the Internet. Today, IoT is moving into virtually every sector, opening new opportunities and new challenges. IoT applications links from smart meters and smart thermostat, to smart grid and smart cities. IoT technology enabled physical objects to make a prompt and better response to environmental changes and make informed decisions (Perera et al. 2014).

IoT has the potential to become one of the most disruptive technologies in decades. IoT enables digital connectivity to devices and sensors to reach almost any places that previously unthinkable. By 2025 , it is predicted there will be 41.6 billion connected IoT devices in the world and generating 79.4 zettabytes (ZB) of data (IDC 2020).

In general, IoT architecture can be structured into threelayered model: perception (sensing), network, and applications layer (Lin et al. 2017; Miao et al. 2010; Zhihong et al. 2011).

\subsubsection{1st layer-perception layer}

Internet-enabled devices from wireless sensors, Radio-frequency identification (RFID), Global Positioning System (GPS), to mobile devices and automobiles are what makes up the perception layer. It is essentially an eco-system of devices that can collect, detect, and exchange information with other devices through different communication networks.

\subsubsection{2nd layer-network layer}

The role of the network layer acts as an intermediary between the perception layer and the application layer. It transports the raw information gathered, via a combination of short-range (ZigBee and Bluetooth) and long-range communication technologies (PLC, Wi-Fi, 3G, 4G, 5G), all dependent on the device's network capability, otherwise known as middleware technology.

\subsubsection{3rd layer-application layer}

Finally, the application layer is the most important for the user as this is where the raw data is received, analyzed and processed to display real time feedback (Talari et al. 2017). Depending on the design of the system, artificial intelligence can provide automated services based on the information given. These systems play an important role in smart cities, to enhance standards of living by incorporating it into services such as, transportation, traffic management and streetlights.

\subsection{Application of Internet of Things}

The effects of accelerated developments and growth problems mentioned in Sect. 1, makes it difficult to maintain the same level of urban living. However, this emerging technological growth of IoT is causing a shift to a more dynamic approach to combat new challenges in the future. Several studies (Hui et al. 2017; Janssen et al. 2019; Kramers et al. 2014; Sánchez et al. 2013) have proposed the idea of incorporating ICT and IoT into cities to improve and drive changes in urban living to become a more sustainable or 'smart' city.

Smart cities are fundamentally much more complex to normal ones (Streitz 2019). A simple definition of a smart cities is a city that uses IoT and ICT to exploit data, to improve government infrastructures and public welfare (Zanella et al. 2014). Whilst the use of technology is important, it also includes changes to many levels of urban living and incorporates many aspects ecologically sustainable lifestyles (Berra and Nuciari 2013), as such, it is also known as a sustainable city. 
In Sánchez et al. (2013), the integration of IoT with traditional utility infrastructures is studied. It mentions the feasibility of implementing new services to existing archetypes in streetlights to provide more efficient public service for the city. Despite the fact that these studies provide evidence of the potential to being more sustainable, there is no guarantee that it is applicable to Sheffield. Every 'smart' solution may or may not be sustainable, depending on the size of its own environmental footprint and on the actual reduction of environmental impact it brings about by improving other processes (Hilty et al. 2014).

Applying the three layers of IoT from Sect. 2.1 in streetlights, allows cities to build a connected lighting network with a central control and monitoring system, in Sheffield a similar system is used called a central management system (CMS).

The implementation of IoT in streetlights, divided into the three stages as represented by the three layers discussed earlier.

\begin{tabular}{cc}
\hline 1st stage_perception layer & Wireless Sensor Networks (WSNs) to \\
monitor data on the environment \\
- Light sensors to monitor the light \\
level \\
- Rain gauge to measure precipitation \\
- Wind direction vane and anemom- \\
eter to measure the wind speed and \\
direction \\
- Water level to measure ground water \\
level for flooding \\
- Environmental air pollution detector \\
measures traffic pollution \\
WSN's able to receive and retransmit \\
data from gateways, ZigBee/WLAN \\
based communication protocol used \\
to communicate between neighboring \\
WSN's, creating a mesh network \\
WSN communicates to other gateways \\
creating a larger network \\
The use of Wi-Fi and GPS to transmit \\
data from a base station to the cloud \\
which can be accessed by a central \\
control and monitoring system \\
Responsive measures can be taken \\
based on the data gathered \\
- Alerting authorities if water level is \\
high \\
- Dimming of streetlights if surround- \\
ing level is high \\
Monitoring traffic based on air pollu- \\
tion levels \\
Monitor air levels for any toxic gas \\
present and will alert authorities if \\
present \\
\hline 3rd stage—application layer \\
\end{tabular}

\subsection{Streetlighting in Sheffield}

Streetlights in Sheffield are currently maintained by a citywide highway maintenance contractor called Streets Ahead, it is currently using Telensa's smart city streetlighting solutions to help maintain the streetlights under a centralized network. Below is the current infrastructure in Sheffield.

\begin{tabular}{lc}
\hline 1st stage_perception layer & Telecell sensor nodes provide \\
& low power metering and Global \\
& Positioning System (GPS) \\
2nd stage_network layer & Ultra-Narrow Band (UNB) base \\
& station provides radio coverage \\
& for up to 5000 sensor nodes. \\
& This has a larger range than most \\
& mesh networks. In addition, have \\
& the advantage of overlapping \\
& coverages for resilience \\
& It has yet to provide other applica- \\
& tions than its main purpose but \\
& still provides economic advan- \\
& tages for the city \\
- Replaces night patrols with & instant fault reporting \\
& Provides fault diagnosis \\
& Reduces repair times
\end{tabular}

The main difference between the proposed study by Mendalka et al. (2010) and the one used in Sheffield is the difference in sensors at the perception level. WSN's tend to only have a battery life of three years whereas Telensa claimed that its streetlight controller called the Telecell can run continuously for 7 years, having up to $4 \times$ less power consumption than mesh networks. Also, due to its GPS capabilities, it is a more robust and resilient system, functioning normally when disconnected from the network (a more suitable system for citywide use).

From their sophisticated control programs, PLANet, Sheffield City Council is now able to showcase a more automated approach to streetlighting to the public. Enabling them to remotely control streetlights, monitor them in real time and detect/diagnose faults automatically, in turn reducing manpower needed for maintenance. Streets Ahead have commented "because of the new system, there is a reduction of up to $65 \%$ of energy usage and carbon footprint." However, Sheffield are only utilizing a portion of Telensa's streetlight control capabilities, only having a timetabled ON/OFF lighting scheme dependent on light levels and the solar calendar. This system can be described as having a 'static behavior', due to the lower level of autonomy and self-adaptation, by not being able to process environmental changes and automatically act accordingly.

\subsection{Energy savings}

These innovative approaches to citywide automation mean that it will be hard to evaluate the energy savings of an adaptive lighting system due to its complexity. Factors such as cost of set-up, behavior in the environment, time of the year would greatly affect the evaluation of it. Realistically, to evaluate a system of this nature a long period of time 
is necessary. Below are case studies of smart streetlighting infrastructure within the UK by Telensa, which gives examples of annual yearly savings to help them combat surging electricity prices and reach target carbon emission goals.

\subsubsection{Edinburgh case study}

Edinburgh City Council have started a projected in 2018 to introduce a smart streetlighting solution. This entails connecting 64,000 streetlights across the city under a centralized system. (nearly the same quantity of streetlights being replaced as Sheffield's). This upgrade was forecasted to reduce its $£ 3.2$ million annually energy expenditure by $50 \%$, and since deployment the figure is more likely to reach $60 \%$. Its projected to save them $£ 54$ million over a 20 -year period (Telensa 2020a).

\subsubsection{Doncaster case study}

Doncaster is one of the largest boroughs in the UK and is located in South Yorkshire right next to Sheffield. They currently house 45,000 smart streetlights which cost them roughly $£ 14.4$ millions of internal government funds. They are currently cutting $25 \%$ of their annual electricity spending which equates to $£ 1.3$ million pounds of total savings.

They have achieved this by doing the following:

- Dimming light levels dependent on external factor.

- Precisely reducing the time streetlights are on during dusk and dawn to achieve better efficiency.

- Adapting power use to deliver constant light output, adapting to the combined effects of gradual LED lumen depreciation and dirt build-up between cleaning intervals.

Doncaster are expected to potentially increase their annual savings by trialing a traffic adaptive lighting infrastructure. At the end of 2018, they implemented this to 178 streetlights from Junction 3 into Doncaster, from this they found out the periods of the day where there is significantly less vehicle movement. Due to this issue they moved the lighting schedule from 20:00-5:00 to 22:00-5:00 which cut the power consumption from 67,087 to $51,474 \mathrm{kWh}$ which results in savings of $19 \%$ in energy spending. This successful trial has presented them with new opportunities to reduce power consumptions in other areas (Telensa 2020b).

\section{Methodologies}

To analyze different models, StreetlightSim (Lau et al. 2014) an open source streetlight simulator, is used to present different lighting schemes. The StreetlightSim has been selected due to it is an open-source and its capability to models both traffic patterns and adaptive networked streetlight. The simulation accuracy of the StreetlightSim also has been validated (Lau et al. 2014). There will be four time-based schemes: Conventional, Dynadimmer, Chronosense and Part-Night which have varying capabilities that will be simulated to present a plethora of solutions for Sheffield's street lighting problem. StreetlightSim uses an adapted module from Vehicle in Network Simulation (VeiNS) (Sommer et al. 2008) framework which allows the combination of OMNeT++ and Simulation of Urban MObility (SUMO), to work in unison to provide a simulated road traffic profile. SUMO is a road traffic simulator which handles the random routes that road users (pedestrians and cars) follow within the simulation and Veins helps with the individual modelling of each user. $\mathrm{OMNeT}++$ allows the simulation of data processing and control algorithms, and communication networks-supporting frameworks and models including MiXiM, INET and INETMANET (OMNet++2020). The relationship between main components is illustrated in Fig. 1. The high-level simulation process flow is shown in Fig. 2. In this figure, step 1 is to obtain a map from www.openstreet map.org. In step 2, we use JOSM to enhance the map by annotating the relative streetlight locations. In step 3, we extract the SUMO road network and streetlight topology from the enhanced map. In step 4, we convert the SUMO random paths and other required configurations to StreetlightSim format. Finally, in step 5 we run the simulation. More detailed information on the StreetlightSim simulator can be found in (Lau et al. 2014).

\subsection{Road traffic model}

To help the simulation generate a realistic evaluation of a streetlight network, it is important to have an accurate road traffic model. A road traffic model has been derived from (DepartmentForTransport 2019) to be able to distribute the annual average daily traffic flow (AADF) of the region throughout the day (per hour). StreetlightSim provides two models of traffic flow as both have different traffic profiles: weekday and weekend. The main difference is that the majority of the population works in the weekday thus will result in higher traffic volumes in the early morning between 7:00 and 9:00 AM. Whereas at the weekend's there is a higher volume of traffic during the middle of the day, between 11:00 and 14:00 (see Fig. 3).

Whilst road users are taken account for with the AADF values given by the government, pedestrian traffic is also important for an accurate evaluation. Eq. (1) shows how pedestrian traffic number is calculated where $\Delta_{\text {ped }}$ is a percentage of the total traffic composition (Lau et al. 2014): 


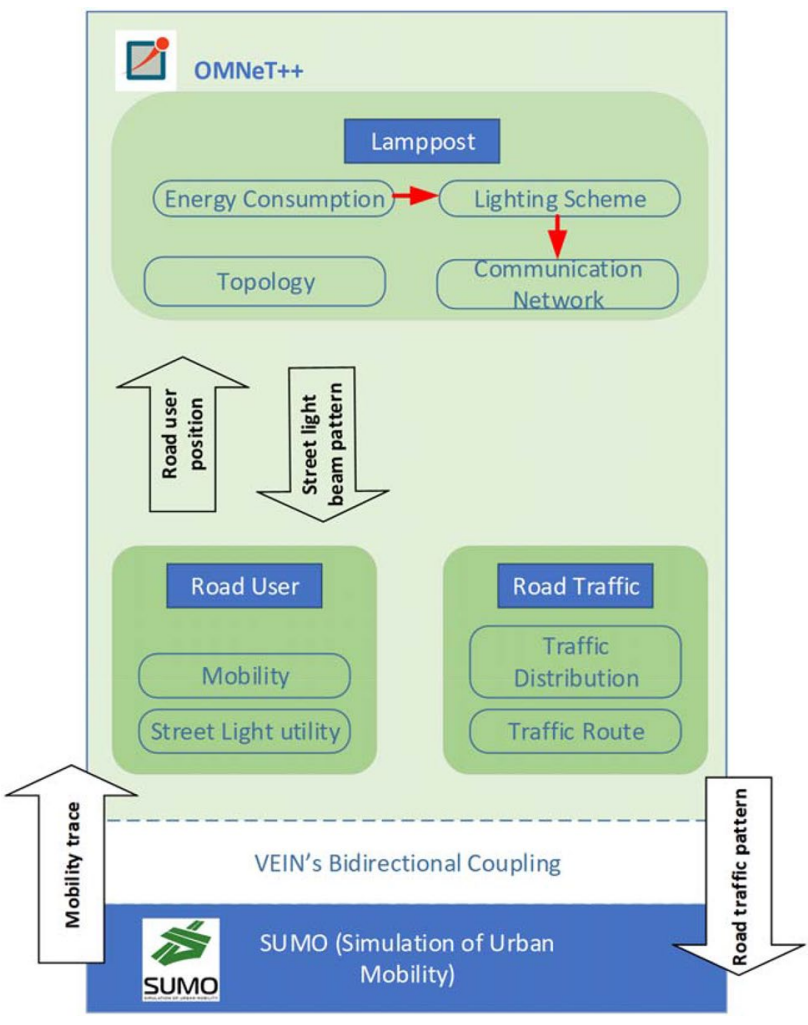

Fig. 1 StreetlightSim Block diagram. (Adapted from Lau et al. 2014)

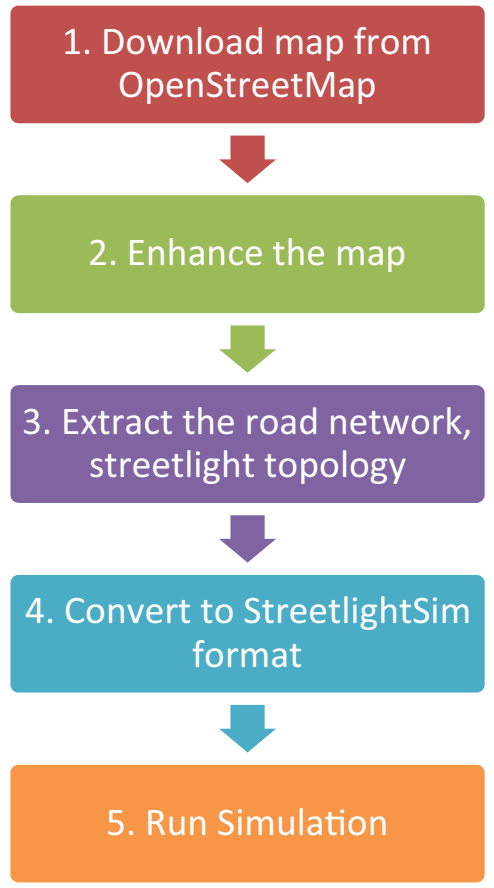

Fig. 2 StreetlightSim high-level process flow. (Adapted from Lau et al. 2014)
$A A D F_{p e d}=\frac{A A D F}{1-\Delta_{p e d}} ; 0 \leq \Delta_{p e d}<1$

Since weekday and weekend have different traffic volumes, the total number of road user injected during the simulation in any given day is given by Lau et al. (2014):

$r(\theta)=\beta(\theta) \times A A D F_{p e d}$

$\theta$ represents weekday or weekend traffic, $\beta$ is the traffic weight of these days. Where is $\mathrm{n}$ StreetlightSim $\beta($ weekday $)=1.13$ and $\beta($ weekday $)=0.87$. Therefore, the total number of road users injected in the simulations, during at hour h, for both weekday and weekend traffic is given by Lau et al. (2014):

$\Gamma(h, \theta)=\omega(h, \theta) \Gamma(\theta)$

where $\omega(\mathrm{h}$,$) is the normalized traffic ratio at hour \mathrm{h}$ on .

\subsection{Lighting scheme}

As mentioned, Sheffield uses a conventional lighting scheme in accordance to the solar clock, however it is trialing dimming schemes in certain regions. Currently, Sheffield procures their streetlights from Signify using products from Phillips-Lighting Luma, Towns guide, and CitySoul range (SheffieldCityCouncil 2020). Phillips Luma range is what you generally see in Sheffield's streets, it is equipped with a power supply and a 'Dynadimmer' for dimming. There are multiple dimming schemes that are available to use in StreetlightSim, each one could be an effective streetlighting solution for Sheffield.

\subsubsection{Dimming scheme}

There are four time-based dimming schemes available in StreetlightSim, each needs to specify the time of the year in terms of months in the configurations (e.g. January month ID $=1$, representing the longest period of lighting

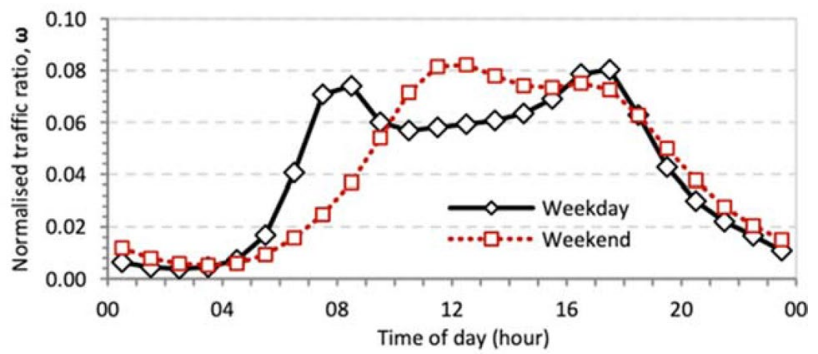

Fig. 3 Traffic distribution ratio by time for the average weekend and weekday vehicular traffic (DepartmentForTransport 2019) 
in the year). They are all derived from existing products or schemes used by other councils.

- Conventional scheme: All streetlights switched on with $100 \%$ brightness until simulation time limit ends.

- Phillips Dynadimmer scheme: Dynadimmers are configurable electronic drivers that has a maximum of 5 set periods of dimming. Dependant on the user, different dimming profiles can be implemented based on the area's geographical location. For this project we will use a typical scheme that is specified in (Phillips 2010), as Sheffield City Council have not disclosed the dimming schedule as its under trial periods. From Fig. 4 we can see the operational hour of this scheme, between 20:00-23:00 it operates at $65 \%, 23: 00-5: 00$ at $40 \%$, and 5:00-6:00 at $55 \%$.

During the rest of its operational hours it operates at 90\% illuminance output of the conventional scheme. This gives an annual energy savings of $40 \%$ if this is implemented.

- Phillips Chronosense scheme: Chronosense is a stand-alone control unit that provide easy installations to newer installations and electromagnetic controlled lights. Unlike Dynadimmer they only provide one step of dimming which the duration can be altered based on its dipswitches (see Fig. 5). This sort of lighting scheme would be effective in more rural or areas where there is little movement during the night. For StreetlightSim it has been configured so between the hours of 22:00 and 5:00, the streetlights will have an illuminance output of
$65 \%$. This equates to a yearly energy savings of approximately 20\% (Phillips 2010).

- Part-night lighting schemes: Part-night lighting have been introduced to other councils such as Warwickshire, Buckinghamshire and Devon. Like the name suggest, this scheme entails streetlights that are being switched off during periods of the night. This solution is very effective in areas where streetlights do not have the ability to be dimmed, or the area in question is isolated. Warwickshire City Council have introduced this to areas where there are no potential hazards nearby, streetlights not operating between 12:00 to 5:00 during the week and 1:00 to 6:30 during the weekends, by doing so have reduced their greenhouse gas emissions by 3000 tonnes, and their annual yearly spending decreasing by $£ 500,000$. StreetlightSim adopts the same lighting scheme (WarwickshireCountyCouncil 2020).

For StreetlightSim to calculate the energy efficiency of different lighting schemes, it assumes that the energy consumed is directionally proportional to its illuminance output. Thus, a model symbolized by the following equation is formed (Lau et al. 2014):

$e(N)=\sum_{n=0}^{N} \operatorname{Pmax} \times \phi T$

where $\mathrm{e}(\mathrm{N})=$ Energy consumed by streetlight after $\mathrm{N}$ discrete timesteps, $\mathrm{N}=$ upper limit of discrete time steps, $\mathrm{n}=$ lower limit of discrete time steps, Pmax $=$ maximum rated power of streetlight and $\phi \mathrm{T}=$ duration of illuminance output by timestep $n$.
Fig. 4 Dynadimmer streetlight operation hours. (Adapted from Phillips 2010)
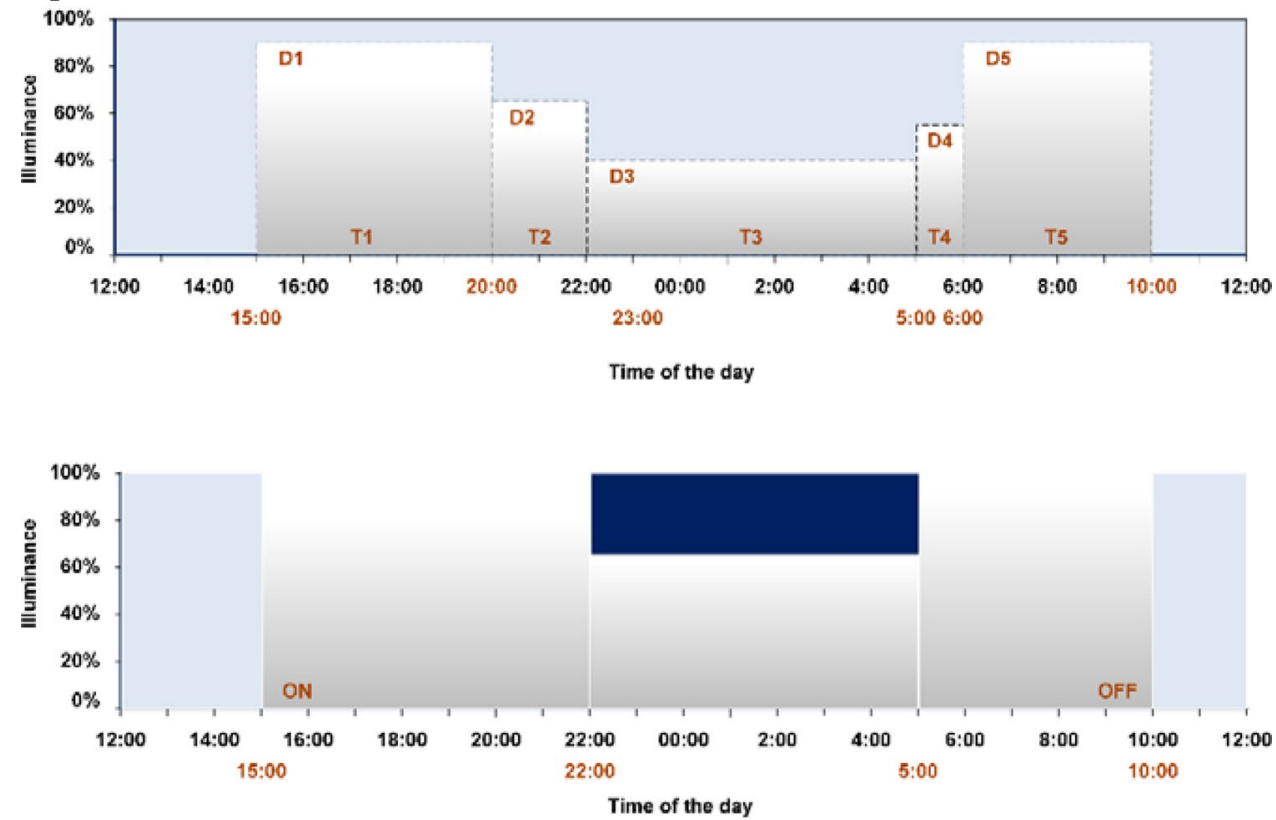

Fig. 5 Chronosense streetlight operation hours. (Adapted from Phillips 2010) 


\subsubsection{Adaptive lighting scheme}

In StreetlightSim there is a proposed adaptive lighting scheme called traffic aware lighting scheme management network (TALiSMaN) (Lau et al. 2015). Instead of using a centralized system to control the light levels of streetlights, it uses an autonomous WSN to detect traffic flow and act upon it. In this scheme, it quantifies the utility of a streetlight by considering different road users (from a pedestrian and motorist perspective). TALiSMaN has four operational states: Lamp on by sensor, Lamp on by neighbour, Lamp on by delay and Lamp off. The state machine of these operation states during operational hours is illustrated in Fig. 7.

From a pedestrian perspective, streetlights are used to navigate through the dark, avoiding any obstacles and identification of other pedestrians by using facial recognition (Davoudian and Raynham 2012; Raynham 2004).

For a pedestrian's perspective it factors in the following:

- How much distance is required to avoid an obstacle.

- The illuminance required for obstacle avoidance, navigation and facial recognition within $10 \mathrm{~m}$.

- Speed of the pedestrian is travelling.

- Pedestrian sense of safety.

Figure 6 illustrates adaptive lighting scheme from a pedestrian perspective (Haans and De Kort 2012). It utilizes descending light distribution for pedestrians, so streetlights within the vicinity have max illuminance output, whilst those further away will decrease in segments. So when the pedestrian is within a streetlight's sensor range [default 13 $\mathrm{m}$ (Yue et al. 2010)], streetlights within a $30 \mathrm{~m}$ distance will have a brightness of $100 \%$. After each $30 \mathrm{~m}$ segment from the pedestrian, the illuminance output will incrementally decrease by $20 \%$. This continues until the distance $\times$ is more than $150 \mathrm{~m}$ and at this point the streetlight is switched off.

The importance of streetlights from a motorist perspective is very high, this is because streetlighting extends the range of which motorist can see beyond their given headlamps,

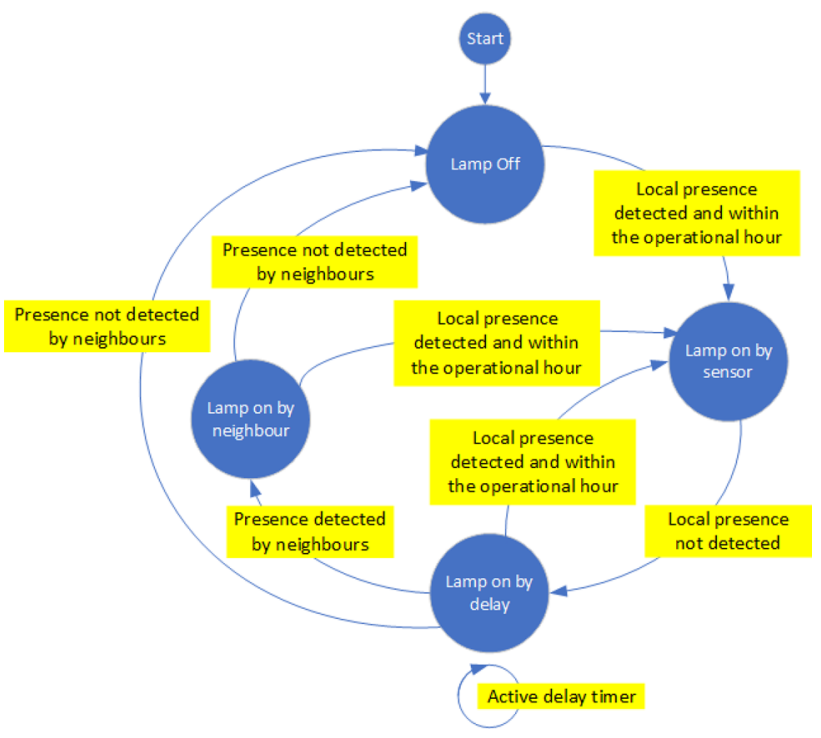

Fig. 7 TALisMaN operation state machine during operational hours. (Adapted from Lau et al. 2015)

which helps them avoid hazards from a distance and gives them time to do an emergency stop if needed. The main difference between a motorist and a pedestrian is the speed at they are travelling, for residential roads the speed limit is typically $30 \mathrm{mph}$ (or $15 \mathrm{~m} / \mathrm{s}$ ) and a pedestrian in average travels at $1.9 \mathrm{~m} / \mathrm{s}$ (Lau et al. 2015).

For a motorist perspective it factors the following:

- Stopping distance of a vehicle based on, vehicle speed, road surface conditions and reaction time.

- Hazard detection.

Figure 8 illustrates adaptive lighting scheme from a motorist perspective. From Fig. 8, we can observe the streetlights S1-S4 having a 100\% illuminance output. This is due to motorist performing better at hazard detection when luminance and uniformity is increased (Brémond et al. 2013; Güler and Onaygil 2003; Mayeur et al. 2010; Staplin 1985).
Fig. 6 Adaptive lighting scheme from a pedestrian perspective. (Adapted from Lau et al. 2015)

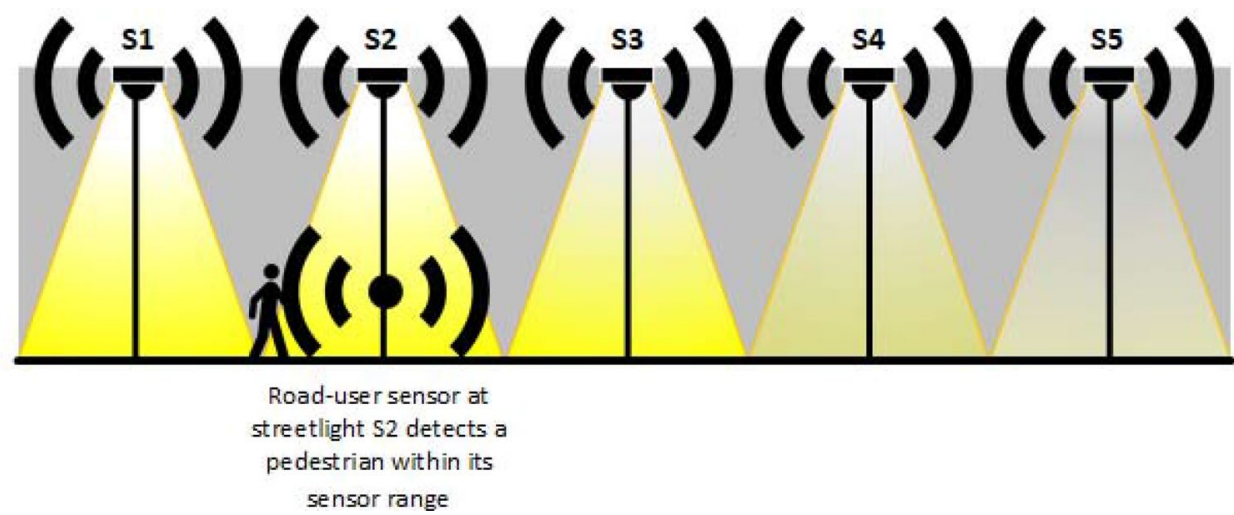


Fig. 8 Adaptive lighting scheme from a motorist perspective. (Adapted from Lau et al. 2015)

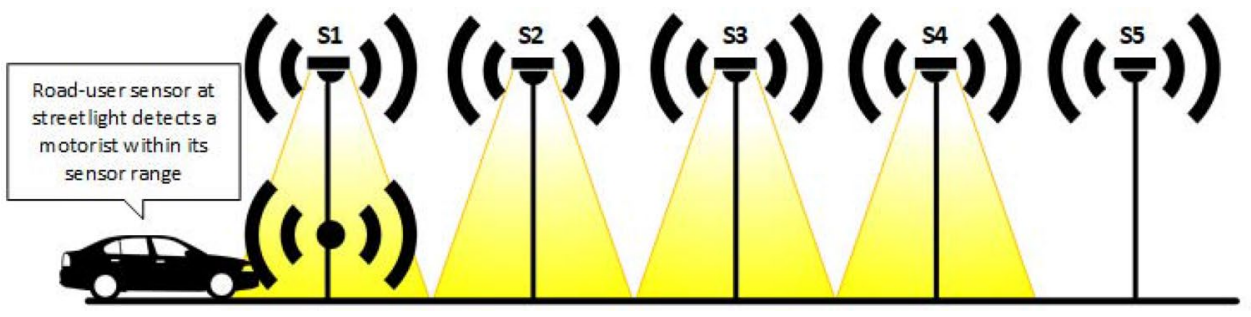

Table 1 Illuminance output of streetlight from motorist and pedestrian perspective (Lau et al. 2015)

\begin{tabular}{lll}
\hline Road user type & Distance from streetlight, d $\begin{array}{l}\text { Illumi- } \\
\text { nance } \\
\text { output }(\%)\end{array}$ \\
\hline Pedestrian & $0 \leq d<30$ & 100 \\
& $30 \leq d<60$ & 80 \\
& $60 \leq d<90$ & 60 \\
$90 \leq d<120$ & 40 \\
& $120 \leq d \leq 150$ & 20 \\
Motorist & $d>50$ & 0 \\
& $0 \leq d \leq 100$ & 100 \\
\hline
\end{tabular}

Also, this model uses the fact it takes a $100 \mathrm{~m}$ to come to a standstill when a hazard has been detected, thus streetlights within this distance, in front of the vehicle is lit up.

This study uses the proposed adaptive scheme for the two roads to be compared to the other dimming schemes presented (Table 1).

\section{Simulation}

\subsection{Simulation parameters}

To ensure the simulations are accurate, the rated power for the streetlights needs to be deduced. From data provided by Street Ahead (Figs. 9, 10), the power consumption for Church Street on the 02/02/2020 was 1147 Wh (see Fig. 10). Thus, we can work out the rated power per streetlight from (Table 2):

$1147 \approx t \times P_{\text {rated }} \times n$

where $\mathrm{t}$ is the duration of streetlight operation $=14 \mathrm{~h}, \mathrm{n}$ is the number of streetlights $=16$.

$P_{\text {rated }} \approx 5.12 \mathrm{~W}$

As it is approximately close to the $5.6 \mathrm{~W}$ value specified in the Luma ranges specification, the study will assume all streetlights to be rated at $5.6 \mathrm{~W}$.
Table 2 Simulation parameters

\begin{tabular}{ll}
\hline Parameter & Value \\
\hline Required lit road segment (motorist) & $100 \mathrm{~m}$ \\
Required lit road segment (pedestrian) & $150 \mathrm{~m}$ \\
Distance between streetlight (average) & $30 \mathrm{~m}$ \\
Operational hours & $17: 00-07.00(14 \mathrm{~h})$ \\
Streetlight power & $5.6 \mathrm{~W}$ \\
AADF & 1900 \\
Sensing range & $13 \mathrm{~m}$ \\
Sensor sampling rate & $20 \mathrm{~Hz}$ \\
Bit rate & $250 \mathrm{kbps}$ \\
Radio propagation model & Simple path loss with \\
& $10 \mathrm{log}-\mathrm{rm}$ al shadowing \\
Minimum bit error rate & effect \\
Radio transmission power & $1 \times 10^{-8}$ \\
\hline
\end{tabular}

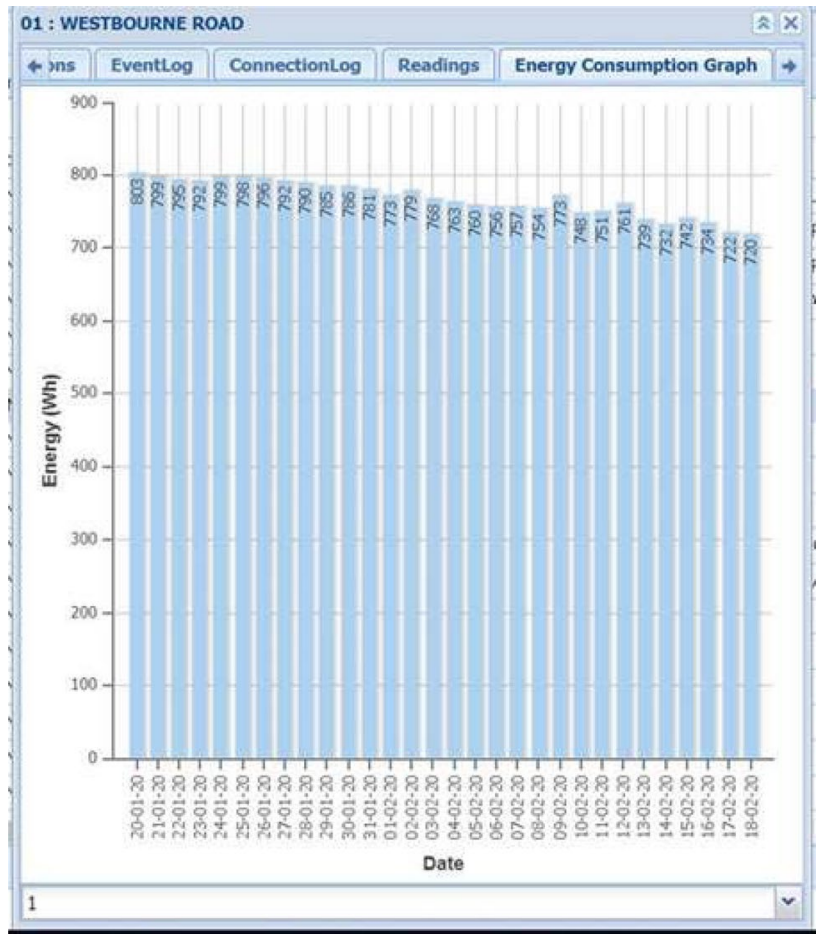

Fig. 9 Westbourne Road real energy consumption (20/01/202018/02/2020) 


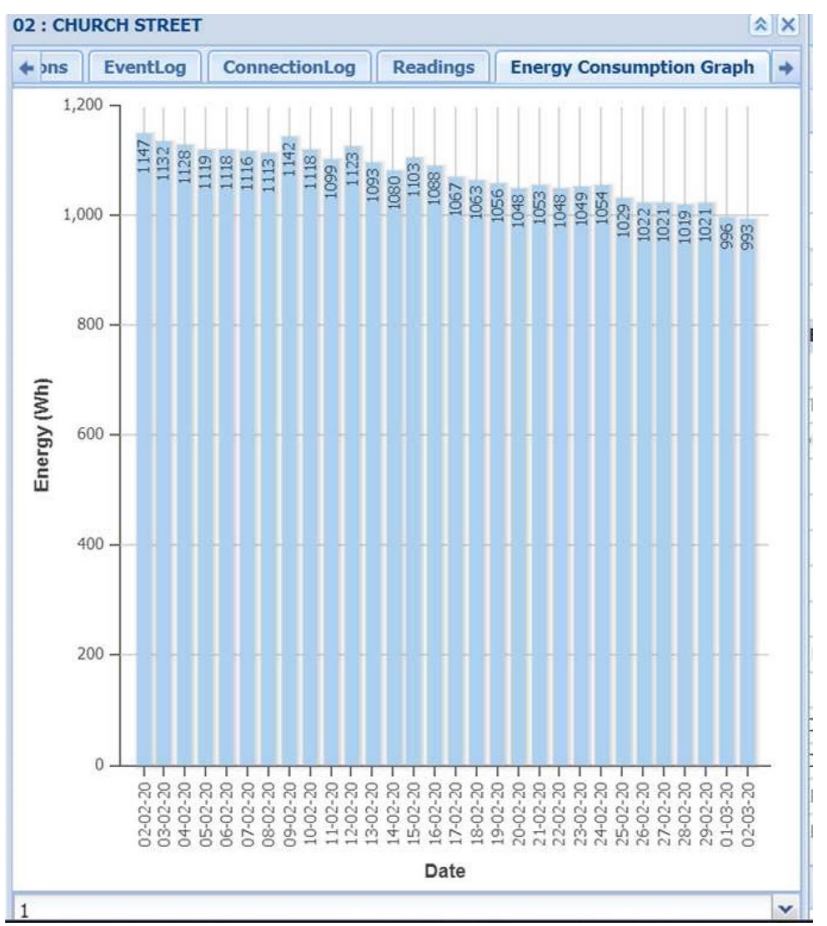

Fig. 10 Church Street real energy consumption (20/02/2020 02/03/2020)

For this study, it evaluates two roads located at different parts of the city (residential and city center). Whilst Sheffield City Council does provide AADF values for Sheffield, it does not provide any for the streets in question. To be able to inject an accurate road traffic profile, AADF values for the region from 'Yorkshire and the Humber' (which Sheffield is under) is taken. Sheffield City is a very populated city. In Transport (2020) there are two values given for minor roads: for rural and for urban. The definition of the urban minor road is that it is within a settlement of 10,000 people or more, and as Sheffield is a major city it easily meets this definition. Thus, we will use the AADF value of 1900, to inject it to both road traffic profiles of the simulations. The simulations will also assume weekday traffic flow, as the data from Streets Ahead for Westbourne Road and Church Street energy consumption in [Wh] is dated from 20/01/2020-18/02/2020 and 02/02/2020-02/03/2020 respectively. To make the simulations more comparable, we use the energy consumption at $02 / 02 / 2020$ for both roads which is equivalent to 14 hours of streetlight operations (17:00-7:00).

\subsection{Westbourne Road}

Westbourne Road is in a residential area. The main reason why this was chosen was because this area was under dimming trials and as energy consumption data was given from the trials, it would serve as a good comparative to conventional lighting schemes.

Using Google street view, it has been identified that this street contains 17 streetlights which is represented by red dots in Fig. 11. It is approximately $1000 \mathrm{~m}$ long road. Note, from the cross section located at the middle of the map to Clarkehouse Road is a one-way system.

\subsection{Church Street}

Like Westbourne Road, Church Street uses a one-way system with approximately $500 \mathrm{~m}$ of straight road. It is located right in the middle of the City and is one of the main reasons why this was chosen to be simulated. With different locations (residential/city center), it will provide the study a wider data range.

Using Google street view, it has been identified that this street contains 16 streetlights which is represented by red dots in Fig. 12.

Note that there are only six red dots in Fig. 12. That is because, the streetlight at the furthest left and right of the map has six light modules in one location as shown in Fig. 13. StreetlightSim does not manage well on placing six streetlights on top of another or right next to each other.

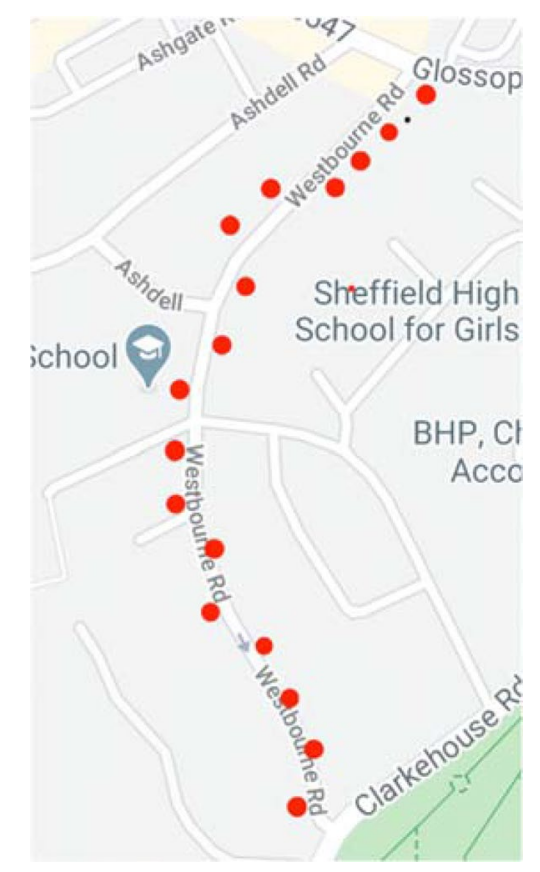

Fig. 11 Westbourne Road streetlight location

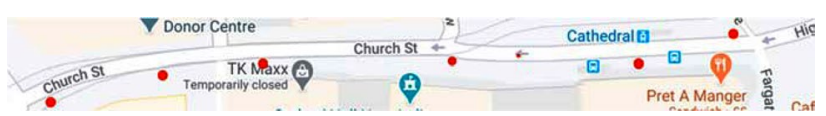

Fig. 12 Church Street streetlight locations 


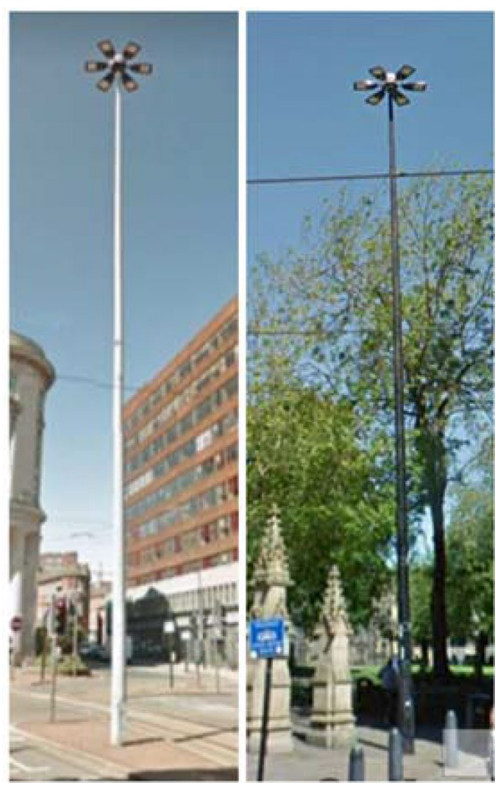

Fig. 13 Church Street streetlights

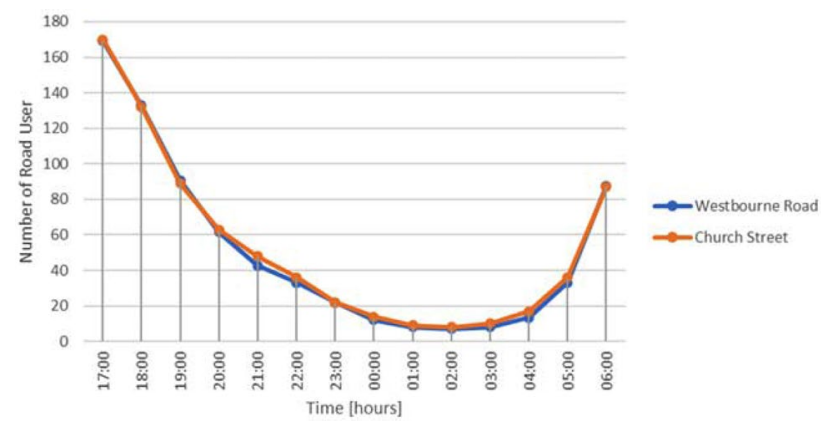

Fig. 14 Traffic flow by road user count for Westbourne Road and Church Street

Therefore, the solution is to use only one streetlight in the simulation, and to calculate its real power consumption. The results then multiplied by six.

\subsection{Results analysis}

The road user count for both simulations is shown in Fig. 14. We notice that both have very similar road traffic profiles for the duration of its streetlight operation, only having minor differences at certain hours. It also follows the trend in the transportation ratio for the weekday shown in Fig. 3.

\subsection{Evaluation of dimming schemes}

As predicted, the lighting scheme with the largest power consumption is the conventional scheme having a value of, 1332.4 and 1254.4 [Wh] for Westbourne Road and Church Street respectively. Comparing the simulated and actual value for Church Street, we can see that there is a minor difference by $8.9 \%$ or 107.4 [Wh] (1254.4 vs. 1147), which is probably caused by not having an exact durations of the streetlight operation hours, as StreetlightSim assumes for the month of February to have operating hours $=14$. Another probable cause is, during sunset and sunrise streetlights gradually switch ON/OFF which would also reduce its power consumption.

From Table 3, we can see that the Chronosense scheme has the second largest power consumption with 1166.2 and 1097.6 [Wh] respectively, which correlates to being approximately $13.3 \%$ energy difference from the conventional scheme. Whilst it does reduce energy spending, it lacks the flexibility it needs for different geographical locations compared to a Dynadimmer scheme, where we can configure it to specific area. Also, as the Chronosense is not equipped to the current streetlights in Sheffield, the council will need to purchase this stand-alone product and install it which would cost large amounts of money and time. Therefore, its environmental benefits do not outweigh the economical and practical disadvantages this lighting scheme has.

Next, both Part Night and Dynadimmer schemes have the same power consumption with 809.2 and 761.6 [Wh] respectively, which has a difference of approximately $48.89 \%$ from the conventional scheme. The value for Westbourne Road has similarities with the real value (see Fig. 9), only having a difference of $3.8 \%$. Therefore, we can say that the current trial dimming schemes is also saving up to $48.89 \%$ energy (however actual lighting scheme may differ). Despite, both Part-Night and Dynadimmer schemes having the same energy expenditure, it would be more beneficial to use the Dynadimmer scheme. This is because, in a Dynadimmer scheme the lights are still switched on throughout the night at varying illuminance output, which maintains the utility of streetlights. Furthermore, as the area of simulations are located in areas of large population (a residential area and
Table 3 Power consumption by lighting scheme for Westbourne Road and Church Street

\begin{tabular}{lllllc}
\hline & $\begin{array}{l}\text { Conven- } \\
\text { tional (Wh) }\end{array}$ & Part Night (Wh) & $\begin{array}{l}\text { Dynadim- } \\
\text { mer }(W h)\end{array}$ & Chronosense (Wh) & Adaptive (Wh) \\
\hline Westbourne Road & 1332.4 & 809.2 & 809.2 & 1166.2 & 75.6 \\
Church Street & 1254.4 & 761.6 & 761.6 & 1097.6 & 143.4 \\
\hline
\end{tabular}


the city center), it is crucial that streetlights are switched on to enhance security and safety in these areas as it is proven to reduce crime rate and encourage socio economics activities during the night (Lau et al. 2014).

The data gained from StreetlightSim for the different dimming features is accurate, as these schemes are time based therefore external factors such as road users and geographical location does not affect the outcome of the result.

\subsection{Evaluation of adaptive schemes}

To create an accurate representation of the adaptive scheme for both road profiles, the simulation has been used five runs each and take an average of it to work out the power consumption. As we can see from Figs. 15 and 16, both have very little differences between the runs, apart from an anomaly found at hour 6:00 in Fig. 15, where Run 1 and Run 4 have a difference of $21 \%$. This is due to different road users at the given time as more pedestrians can cause bigger power consumption, or the route generated goes pass more streetlights which causes the same result. However, overall, both figures follow a similar trend to Figs. 3 and 14 , as power consumption is proportional to traffic flow in an adaptive scheme. Although both have similar trends, the key difference between the two is that, Church Street has a larger power consumption with 143.4 [Wh] compared to Westbourne Road's 75.6 [Wh], despite having one less streetlight. The most likely cause for this situation, is probably the streetlight vs. road length density, as Westbourne Road is approximately double the length of Church Street with $1000 \mathrm{~m}$, which equates to a streetlight roughly every 59 $\mathrm{m}$, compared to one every $31 \mathrm{~m}$. Another factor is the higher complexity of Westbourne Roads streets, as there are more paths that can be taken whereas, Church Street is a simple one-way road, so the likelihood of all the streetlights activating by one road user is very high.

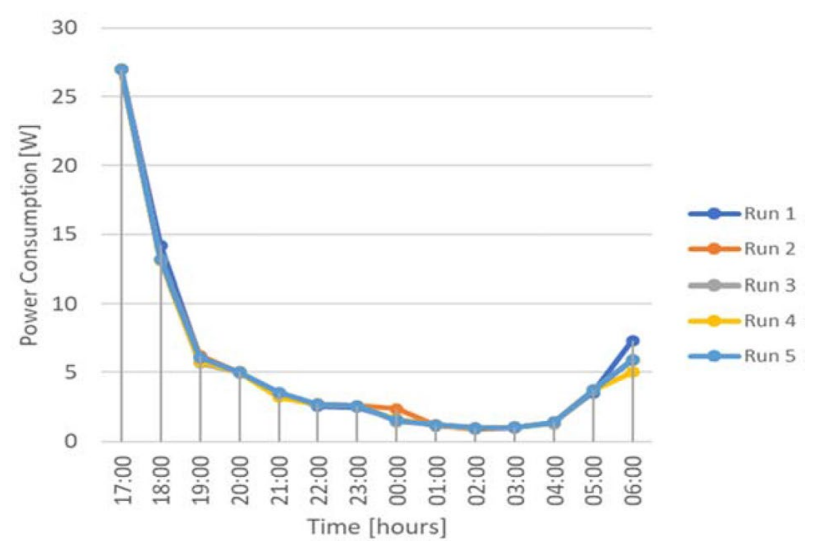

Fig. 15 Westbourne Road adaptive power consumption

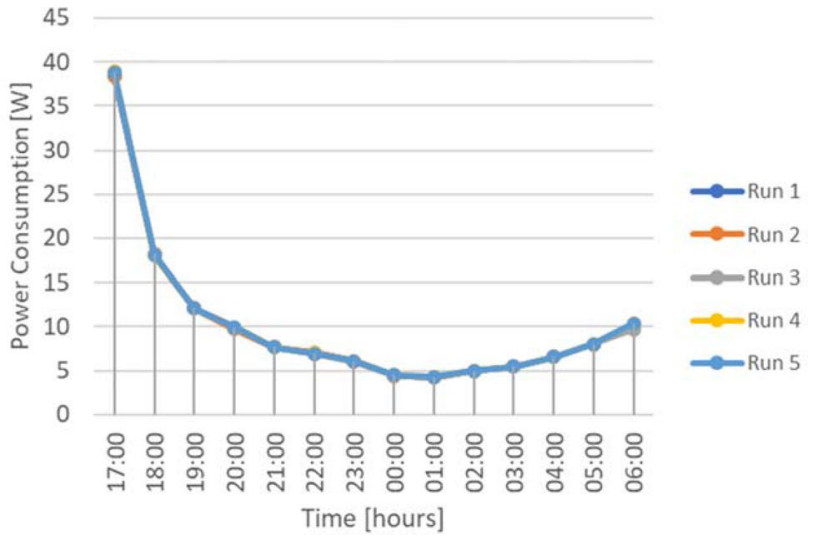

Fig. 16 Church Street adaptive power consumption

A big standout is in Table 3 where we can see a major difference in power consumption compared to any of the dimming and the conventional schemes, having up to a difference of 1256.8 [Wh] which equal to $0.293 \mathrm{~kg} \mathrm{CO} 2$ or $179 \%$ difference in energy expenditure. The majority of its savings is during the times when there is low traffic (between 22:00-05:00), where the lowest road user value is only eight. In essence, the proposed TALiSMaN adaptive scheme is most effective in residential areas where there are multiple pathways and longer roads, compared to a busier city center road such as Church Street. However, despite showing great results in terms of reduction in energy expenditure, there are flaws to this scheme. Firstly, as mentioned, different pathways for road users can be taken, StreetlightSim randomly generate these pathways which means it is not necessarily the correct road traffic flow for Sheffield. Another problem is the AADF value, the value 1900 was taken from a more generic region, the 'Yorkshire \& Humber' which Sheffield is under. However, this takes into account other towns and cities that have smaller populations, consequently, we can say that the result from the adaptive scheme is not accurate to Sheffield but for urban minor roads in 'Yorkshire \& Humber' region. For that reason, more comprehensive research is needed in Sheffield to be able to full predict the outcome of the proposed adaptive scheme.

\subsection{Energy savings}

Based on Department for Business, Energy and Industrial Strategy, the average price for electricity per $\mathrm{kWh}$ is 12.9 pence (p) (BEIS 2020). This figure will be used to calculate energy expenditure for each lighting scheme (see Table 4).

When averaged per hour it works out to be (see Table 5):

If we assume streetlight operation for each day in each month is the same, i.e. the days in January $=16 \mathrm{~h}$, February $=14 \mathrm{~h}$, March $=12 \mathrm{~h}$, April $=10 \mathrm{~h}$, May $=8 \mathrm{~h}$, June $=8$ h, July $=8$ h, August $=10$ h, September $=12$ h, October $=13$ 
hs, November $=15 \mathrm{~h}$, December $=16 \mathrm{~h}$ (from Conventional streetlighting by month scheme in StreetlightSim). We get the total hours of streetlight operations $=4315 \mathrm{~h}$. Thus, the cost per year for each scheme can be calculated, represented in Table 6.

From Table 6, the largest energy saving with $178 \%$ from the conventional scheme is by using an adaptive approach. However as mentioned in Sect. 4.6, using this scheme is inconclusive without using more accurate representation of Sheffield road traffic flow and AADF values. Hence, the most reliable scheme for this moment, is using a Dynadimmer scheme, which can save Sheffield City Council up to $49 \%$ of energy expenditure. The comparison of the level of energy-savings with other smart streetlights proposals is discussed in the Sect. 5 section.

\section{Related work}

Smart streetlights have been implemented all around the world due to its benefits and advantages. In this section, key points of related smart streetlights works are highlighted and energy-savings are compared (if applicable).

In Müllner and Riener (2011), an energy efficient pedestrian aware smart street lighting (SSL) system based on Global Positioning System (GPS) and Internet enabled mobile phone is proposed. The GPS signal from pedestrian is used to turn ON/OFF streetlights. ZigBee protocol is used to communicate between streetlights. However, no real comparison with other similar systems is presented.

Leccese (2013) proposed intelligent LED streetlights based on remote-control. The proposal implemented solar panels to save energy and used ZigBee devices, sensors, and protocol for street-lamp-system management. In the paper, the level of energy-savings is not clearly mentioned.

An autonomous intelligent LED streetlight system which considers vehicle and pedestrian based on environmental sensors is proposed by Elejoste et al. (2013). The prosed system implemented 3-level hierarchical model: end nodes, remote concentrators, and remote-control system. ZigBee is used for communication between nodes. The paper claimed to have on average around $40 \%$ energy-savings. The paper also proposed an improvement called adaptive neuro-fuzzy inference system (ANFIS) to improve scalability and fault tolerance.

Leccese et al. (2014) used ZigBee sensors network and WiMAX to develop full control smart street lighting in smart city application (Leccese et al. 2014). Raspberry Pi is adopted to give the coordinator lamp post a computing power needed. The proposed system uses local sensors for intelligent lighting of the lamp. From the paper, the level of energy-savings is not clearly mentioned.

Smart and energy-efficient LED streetlight system using ZigBee network is proposed by authors in (Kaleem et al. 2014). The proposed system used ZigBee mesh network for communication with streetlights, gateway node, and management software for real-time monitoring and control. Light sensors are implemented to adjust the illumination level of the streetlights. The paper claimed that the proposed system has the capability to reduce power consumption, but no level of energy-savings is mentioned.

A distributed Traffic-Aware Lighting Scheme Management Network (TALiSMaN) system is proposed by Lau et al. (2015). The proposed system uses WSN and considers pedestrian and motorist traffic. The StreetLightSim is used to evaluate the proposal. Up to $45 \%$ reduced energy savings

Table 4 Energy savings for $02 / 02 / 2020$

\begin{tabular}{llllll}
\hline & Conventional & Part Night & Dynadimmer & Chronosense & Adaptive \\
\hline Westbourne Road & $1332.4 \times 12.9=17.19 \mathrm{p}$ & $809.2 \times 12.9=10.44 \mathrm{p}$ & $809.2 \times 12.9=10.44 \mathrm{p}$ & $1166.2=15.04 \mathrm{p}$ & $75.6=0.98 \mathrm{p}$ \\
Church Street & $1254.4 \times 12.9=16.2$ & $761.6 \times 12.9=9.82 \mathrm{p}$ & $761.6 \times 12.9=9.82 \mathrm{p}$ & $1097.6 \times 12.9=14.16 \mathrm{p}$ & $143.4 \times 12.9=1.85 \mathrm{p}$ \\
\hline
\end{tabular}

Table 5 Average price per hour

\begin{tabular}{llllll}
\hline & $\begin{array}{l}\text { Conven- } \\
\text { tional (pence) }\end{array}$ & $\begin{array}{l}\text { Part } \\
\text { Night (pence) }\end{array}$ & $\begin{array}{l}\text { Dynadim- } \\
\text { mer (pence) }\end{array}$ & $\begin{array}{l}\text { Chron- } \\
\text { osense (pence) }\end{array}$ & $\begin{array}{l}\text { Adap- } \\
\text { tive (pence) }\end{array}$ \\
\hline Westbourne Road & 1.23 & 0.75 & 0.75 & 1.07 & 0.07 \\
Church Street & 1.15 & 0.7 & 0.7 & 1.01 & 0.13 \\
\hline
\end{tabular}

Table 6 Average cost per year

\begin{tabular}{|c|c|c|c|c|c|}
\hline & Conventional (f) & Part Night (£) & Dynadimmer $(\mathfrak{f})$ & Chronosense (f) & Adaptive $(\mathfrak{f})$ \\
\hline Westbourne Road & 53.07 & 32.36 & 32.26 & 46.17 & 3.02 \\
\hline Church Street & 49.62 & 30.21 & 30.21 & 43.58 & 5.61 \\
\hline
\end{tabular}


is achievable (depending on traffic volume) compared to multi-sensor method.

Shahzad et al. (2016) proposed energy-efficient intelligent LED street lighting system by implementing traffic-adaptive control. The authors used low power ZigBee mesh network to communicate between different streetlight nodes. The flow of traffic is shared between nodes to collect the density of people to adjust the intensity of LED streetlights. The paper claimed that the proposal was able to save $68-82 \%$ energy depending on the variations in daylight hours between summer and winter (Shahzad et al. 2016).

Bellido-Outeiriño et al. (2016) proposed a smart streetlights system based on Digital Addressable Lighting Interface (DALI) protocol and IEEE 802.15.4 for communications and controlling the streetlights (Bellido-Outeiriño et al. 2016). The proposed system claimed to have energy-saving around $30-40 \%$ compared to other system that used astronomical time relays (ATRs) or double-level ballast (DLB).

Smart LED streetlight system for smart city with webbased management system has been studied in Daely et al. (2017). They used ZigBee, 3G and IP-based technologies for communications. TCP/IP is used to control LED lamps. The level of energy-savings is not mentioned.

An artificial neural network (ANN) based energy efficiency smart street lighting systems is proposed by Mohandas et al. (2019). The proposed system was evaluated in a residential area. Five different scenarios were considered. ANN and fuzzy logic controller were implemented to help make an efficient decision making. The work reported to achieve $13.5 \%$ reduction in energy consumption.

Bingol et al. proposed an adaptive LoRa-based smart streetlighting system for smart cities in Bingöl et al. (2019). The central unit (alarm system, persistence layer and control unit) collects data from and delivers commands to the nodes (LED with an embedded controller) to adjust their illumination level. The level of energy-savings is not mentioned (Table 7).

\section{Summary}

Streetlights are one of the biggest contributors to energy expenditure in the UK which amounts to approximately $1874 \mathrm{GWh}$ with the current electricity rates increasing as each year goes by, there is a need to reduce power consumption by introducing new schemes. The main aim for this study was to introduce IoT in Sheffield to reduce energy consumption in streetlights, to fully meet this aim several conditions was needed to be met in forms of objectives to be completed.

Firstly, compared to Sheffield's neighbors, Doncaster, there has not been much development in using more adaptive and autonomous methods of energy saving. Whilst, Sheffield have successfully implemented the Streets Ahead program to utilize a central management system, they are not taking advantage of the Telensa System fully.

On the other hand, they have recently started implementing different dimming schemes to certain areas, the results gained from StreetlightSim for the different schemes are encouraging to see, having energy savings up to $178 \%$ for the adaptive scheme proposed in Sect. 4.6. At first, this may seem like a huge savings but if this scheme was implemented in Sheffield now, the results may vary depending on the following factors:

- Location of proposed scheme.

- Type of Streetlights.

- Type of road.

As this scheme needs to be further evaluated, the percentage energy saved figure is unreliable. Despite this setback, it is however, encouraging to see the results of the other dimming schemes, in particular the Dynadimmer scheme. The majority of Sheffield's streetlights uses the Luma range which happens to be equipped with the Dynadimmer driver, it concluded that it was the most reliable scheme as it saved nearly $50 \%$ of energy compared to a conventional scheme. Furthermore, the Part-Night lighting scheme have also produced the same result, meaning, this could be possibly implemented in more isolated areas such as the $0.5 \%$ of Sheffield's current streetlights that have yet to be replaced. This would be an effective solution to other streetlights that do not have the dimming capabilities as the Phillip Lighting Luma range.

In conclusion, the study does present an IoT embedded solution for streetlights to reduce energy consumption, it has shown different lighting schemes resulting in different reduction in energy consumption, whilst the adaptive approach remains inconclusive, it does present an obvious reduction in power consumption based on the relationship between traffic flow and total energy expenditure. The solution can be applicable to other cities in reducing energy-consumption. 
Table 7 Summary of related work

\begin{tabular}{|c|c|c|}
\hline References & Key points & Control method \\
\hline Müllner and Riener (2011) & $\begin{array}{l}\text { - Used Global Positioning System (GPS) and Internet enabled mobile phone } \\
\text { - Zoning concept }\end{array}$ & Autonomous \\
\hline Leccese (2013) & $\begin{array}{l}\text { - Intelligent LED streetlight based on remote control } \\
\text { - Solar panels is used to save energy } \\
\text { - ZigBee }\end{array}$ & Adaptive \\
\hline Elejoste et al. (2013) & $\begin{array}{l}\text { - Considered vehicle and pedestrian based on environmental sensors } \\
\text { - Use an adaptive neuro-fuzzy inference system (ANFIS) } \\
\text { - Around } 40 \% \text { energy-savings is possible }\end{array}$ & Autonomous \\
\hline Kaleem et al. (2014). & $\begin{array}{l}\text { - Autonomous smart energy-efficient streetlights system based on ZigBee network } \\
\text { - Used light sensors to adjust the illumination leve }\end{array}$ & Autonomous \\
\hline Leccese et al. (2014) & $\begin{array}{l}\text { - Fully controlled street Lighting based on Raspberry Pi } \\
\text { - Use ZigBee sensors network, and WiMAX }\end{array}$ & Autonomous \\
\hline Lau et al. (2015) & $\begin{array}{l}\text { - A distributed Traffic-Aware Lighting Scheme Management Network (TALiSMaN) } \\
\text { - Used WSN to communicate } \\
\text { - Considered pedestrian and motorist traffic } \\
\text { - Up to } 45 \% \text { energy-savings (depending on traffic volume) compared to multi-sensor method }\end{array}$ & Adaptive \\
\hline Shahzad et al. (2016) & $\begin{array}{l}\text { - ZigBee network is established between different Street Light Nodes } \\
\text { - The flow of traffic is shared between nodes to collect the density of people to adjust the } \\
\text { illumination level } \\
\text { - An energy-savings of } 68-82 \% \text { is possible depending on the variations in daylight hours } \\
\text { between summer and winter }\end{array}$ & Adaptive \\
\hline Bellido-Outeiriño et al. (2016) & $\begin{array}{l}\text { - Used Digital Addressable Lighting Interface (DALI) protocol and IEEE } 802.15 .4 \text { for com- } \\
\text { munications and controlling the streetlights } \\
\text { - Claimed to have energy-saving around 30-40\% compared to other system that used astro- } \\
\text { nomical time relays (ATRs) or double-level ballast (DLB) }\end{array}$ & Adaptive \\
\hline Marino et al. (2017) & $\begin{array}{l}\text { - Used camera network to adaptively monitor the streetlights network } \\
\text { - Control strategy based on traffic forecast } \\
\text { - Results from test bed showed that the proposed system has high potential energy savings }\end{array}$ & Adaptive \\
\hline Daely et al. (2017) & $\begin{array}{l}\text { - Smart streetlights system with web-based central management system } \\
\text { - Considered weather conditions such as fog or pollution } \\
\text { - Used ZigBee, 3G and IP-based communication networks. TCP/IP is used to control LED } \\
\text { lamps }\end{array}$ & Adaptive \\
\hline Mohandas et al. (2019) & $\begin{array}{l}\text { - Five different scenarios were considered } \\
\text { - ANN and fuzzy logic controller were implemented to help make an efficient decision making } \\
\text { - Achieved } 13.5 \% \text { reduction in energy consumption }\end{array}$ & Autonomous \\
\hline Bingöl et al. (2019). & $\begin{array}{l}\text { - Used LoRa communication network } \\
\text { - The central unit (alarm system, persistence layer and control unit) collects data from and } \\
\text { delivers commands to the nodes (LED with an embedded controller) to adjust their illumina- } \\
\text { tion level }\end{array}$ & Adaptive \\
\hline
\end{tabular}

Open Access This article is licensed under a Creative Commons Attribution 4.0 International License, which permits use, sharing, adaptation, distribution and reproduction in any medium or format, as long as you give appropriate credit to the original author(s) and the source, provide a link to the Creative Commons licence, and indicate if changes were made. The images or other third party material in this article are included in the article's Creative Commons licence, unless indicated otherwise in a credit line to the material. If material is not included in the article's Creative Commons licence and your intended use is not permitted by statutory regulation or exceeds the permitted use, you will need to obtain permission directly from the copyright holder. To view a copy of this licence, visit http://creativecommons.org/licenses/by/4.0/.

\section{References}

Ashton K (2009) That 'Internet of Things' thing. RFID J 22:97-114 Atzori L, Iera A, Morabito G (2010) The Internet of Things: a survey. Comput Netw 54:2787-2805

Badgelwar SS, Pande HM (2017) Survey on energy efficient Smart Street Light system. In: 2017 international conference on I-SMAC (IoT in Social, Mobile, Analytics and Cloud) (I-SMAC). IEEE, pp 866-869

BEIS (2020) Gas and electricity prices in the non-domestic sector. https://www.gov.uk/government/statistical-data-sets/gas-and-elect ricity-prices-in-the-non-domestic-sector. Accessed 1 June 2020 
Bellido-Outeiriño FJ, Quiles-Latorre FJ, Moreno-Moreno CD, FloresArias JM, Moreno-García I, Ortiz-López M (2016) Streetlight control system based on wireless communication over DALI protocol. Sensors 16:597

Berra M, Nuciari M (2013) Smart cities. ICT infrastructures for social participation? Quaderni di Sociologia 63(63):127-153. https:// doi.org/10.4000/qds.427

Bingöl E, Kuzlu M, Pipattanasompom MA (2019) LoRa-based Smart Streetlighting system for Smart Cities. In: 2019 7th international Istanbul Smart Grids and Cities Congress and Fair (ICSG), 25-26 April 2019, pp 66-70. https://doi.org/10.1109/SGCF.2019.87824 13

Borgia E (2014) The Internet of Things vision: key features, applications and open issues. Comput Commun 54:1-31. https://doi. org/10.1016/j.comcom.2014.09.008

Boulaalam A (2019) Internet of Things: new classification model of intelligence. J Ambient Intell Human Comput 10:2731-2744. https://doi.org/10.1007/s12652-018-0965-2

Brémond R, Bodard V, Dumont E, Nouailles-Mayeur A (2013) Target visibility level and detection distance on a driving simulator. Light Res Technol 45:76-89

Daely PT, Reda HT, Satrya GB, Kim JW, Shin SY (2017) Design of Smart LED Streetlight system for Smart City with web-based management system. IEEE Sens J 17:6100-6110. https://doi. org/10.1109/JSEN.2017.2734101

Davoudian N, Raynham P (2012) What do pedestrians look at at night? Light Res Technol 44:438-448

DepartmentForTransport (2019) Traffic distribution on all roads by time of day in Great Britain. https://www.gov.uk/government/stati stical-data-sets/road-traffic-statistics-tra. Accessed 31 May 2020

Elejoste P et al (2013) An easy to deploy Street Light control system based on wireless communication and LED technology. Sensors 13:6492-6523

Escolar S, Carretero J, Marinescu M-C, Chessa S (2014) Estimating energy savings in Smart Street Lighting by using an adaptive control system. Int J Distrib Sens Netw 10:971587

Feki MA, Kawsar F, Boussard M, Trappeniers L (2013) The Internet of Things: the next technological revolution. Computer 46:2425. https://doi.org/10.1109/MC.2013.63

Gubbi J, Buyya R, Marusic S, Palaniswami M (2013) Internet of Things (IoT): a vision, architectural elements, and future directions. Future Gener Comput Syst 29:1645-1660. https://doi. org/10.1016/j.future.2013.01.010

Güler Ö, Onaygil S (2003) The effect of luminance uniformity on visibility level in road lighting. Light Res Technol 35:199-213

Haans A, De Kort YA (2012) Light distribution in dynamic street lighting: two experimental studies on its effects on perceived safety, prospect, concealment, and escape. J Environ Psychol 32:342-352

Hilty LM, Aebischer B, Rizzoli AE (2014) Modeling and evaluating the sustainability of smart solutions . Environ Model Softw 56:15. https://doi.org/10.1016/j.envsoft.2014.04.001

Hui TK, Sherratt RS, Sánchez DD (2017) Major requirements for building Smart Homes in Smart Cities based on Internet of Things technologies. Future Gener Comput Syst 76:358-369

IDC (2020) The growth in connected IoT devices is expected to generate $79.4 \mathrm{ZB}$ of data in 2025. https://www.idc.com/getdo c.jsp?containerId=prUS45213219. Accessed 31 May 2020

Janssen M, Luthra S, Mangla S, Rana NP, Dwivedi YK (2019) Challenges for adopting and implementing IoT in smart cities: an integrated MICMAC-ISM approach. Int Res 29:1589-1616

Javed F, Afzal MK, Sharif M, Kim BS (2018) Internet of Things (IoTs) operating systems support, networking technologies, applications, and challenges: a comparative review. IEEE Commun Surv Tutor PP:1-1. https://doi.org/10.1109/COMST.2018.2817685
Kaleem Z, Ahmad I, Lee C (2014) Smart, energy efficient LED Street light control system using ZigBee network. In: 12th international conference on frontiers of information technology, 17-19 Dec 2014, pp 361-365. https://doi.org/10.1109/FIT.2014.74

Kramers A, Höjer M, Lövehagen N, Wangel J (2014) Smart sustainable cities - exploring ICT solutions for reduced energy use in cities. Environ Model Softw 56:52-62

Lau SP, Merrett GV, Weddell AS, White NM (2014) StreetlightSim: a simulation environment to evaluate networked and adaptive street lighting. In: IEEE Asia Pacific conference on wireless and mobile, 28-30 Aug. 2014, pp 66-71. https://doi.org/10.1109/ APWiMob.2014.6920267

Lau SP, Merrett GV, Weddell AS, White NM (2015) A traffic-aware street lighting scheme for Smart Cities using autonomous networked sensors. Comput Electr Eng 45:192-207. https://doi. org/10.1016/j.compeleceng.2015.06.011

Leccese F (2013) Remote-control system of high efficiency and intelligent street lighting using a ZigBee network of devices and sensors. IEEE Trans Power Deliv 28:21-28. https://doi.org/10.1109/ TPWRD.2012.2212215

Leccese F, Cagnetti M, Trinca D (2014) A smart city application: a fully controlled street lighting isle based on Raspberry-Pi card, a ZigBee sensor network and WiMAX. Sensors 14:24408-24424

Lin J, Yu W, Zhang N, Yang X, Zhang H, Zhao W (2017) A survey on Internet of Things: architecture, enabling technologies, security and privacy, and applications . IEEE Int\&nbsp;Things J 4:11251142. https://doi.org/10.1109/JIOT.2017.2683200

Liu G (2014) Sustainable feasibility of solar photovoltaic powered street lighting systems. Int J Electr Power Energy Syst 56:168-174

Marino F, Leccese F, Pizzuti S (2017) Adaptive street lighting predictive control . Energy Procedia 111:790-799. https://doi. org/10.1016/j.egypro.2017.03.241

Masoud MI (2015) Street lighting using solar powered LED light technology: Sultan Qaboos University case study. In: 2015 IEEE 8th GCC conference and exhibition, 1-4 Feb. 2015, pp 1-6. https://doi.org/10.1109/IEEEGCC.2015.7060015

Mayeur A, Bremond R, Bastien JC (2010) Effects of the viewing context on target detection. Implications for road lighting design. Appl Ergon 41:461-468

Mendalka M, Gadaj M, Kulas L, Nyka K (2010) WSN for intelligent street lighting system. In: 2010 2nd international conference on information technology (2010 ICIT). IEEE, pp 99-100

Miao W, Ting-Jie L, Fei-Yang L, Jing S, Hui-Ying D (2010) Research on the architecture of Internet of Things. In: 3rd international conference on advanced computer theory and engineering (ICACTE), 20-22 Aug. 2010, pp V5-484-V485-487. https:// doi.org/10.1109/ICACTE.2010.5579493

Mohandas P, Dhanaraj JSA, Gao X-Z (2019) Artificial Neural Network based Smart and Energy Efficient Street Lighting system: a case study for residential area in Hosur. Sustain Cities Soc 48:101499. https://doi.org/10.1016/j.scs.2019.101499

Müllner R, Riener A (2011) An energy efficient pedestrian aware Smart Street Lighting system. Int J Pervas Comput Commun 7(2):147-161. https://doi.org/10.1108/17427371111146437

OMNet++ (2020) OMNeT++ network simulation framework. http:// www.omnetpp.org. Accessed 31 May 2020

Perera C, Zaslavsky A, Christen P, Georgakopoulos D (2014) Context aware computing for the Internet of Things: a survey. IEEE Commun Surv Tutor 16:414-454. https://doi.org/10.1109/ SURV.2013.042313.00197

Phillips (2010) The Dynadimmer and the Chronosense. https://www. docs.lighting.philips.com/en_gb/oem/download/dynadimmer /P_Leaflet_Dynadimmer_V2Finpages.pdf. Accessed 31 May 2020

Raynham P (2004) An examination of the fundamentals of road lighting for pedestrians and drivers. Light Res Technol 36:307-313 
Sánchez L, Elicegui I, Cuesta J, Muñoz L, Lanza J (2013) Integration of utilities infrastructures in a future internet enabled smart city framework. Sensors 13:14438-14465

Shahzad G, Yang H, Ahmad AW, Lee C (2016) Energy-efficient intelligent Street Lighting system using traffic-adaptive control. IEEE Sens J 16:5397-5405. https://doi.org/10.1109/ JSEN.2016.2557345

SheffieldCityCouncil (2020) Streets ahead. https://www.sheffield. gov.uk/streetsahead. Accessed 1 June 2020

Sommer C, Yao Z, German R, Dressler F (2008) Simulating the influence of IVC on road traffic using bidirectionally coupled simulators. In: IEEE INFOCOM workshops 2008. IEEE, pp 1-6

Staplin L (1985) Nighttime hazard detection on freeways under alternative reduced lighting conditions. In: Proceedings of the human factors society annual meeting, 1985. vol 7. SAGE Publications Sage CA, Los Angeles, CA, pp 725-729

Streitz N (2019) Beyond 'smart-only' cities: redefining the 'smarteverything' paradigm. J Ambient Intell Human Comput 10:791812. https://doi.org/10.1007/s12652-018-0824-1

Talari S, Shafie-Khah M, Siano P, Loia V, Tommasetti A, Catalão JP (2017) A review of smart cities based on the Internet of Things concept. Energies 10:421

Telensa (2020a) The City of Edinburgh Council: case study. https ://info.telensa.com/case-study-the-city-of-edinburgh-council. Accessed 31 May 2020

Telensa (2020b) Doncaster Council: case study. https://info.telen sa.com/hubfs/Resources\%20page \%20files/doncaster-counc il-annual-energy-savings-of-1.3-million.pdf. Accessed 31 May 2020

UnitedNations (2019) World urbanization prospects-the 2018 revision. United Nations. https://population.un.org/wup/Publications/ Files/WUP2018-Report.pdf. Accessed 08 June 2020

WarwickshireCountyCouncil (2020) Street lighting Part-Night operation frequently asked questions. https://apps.warwickshire.gov.uk/ api/documents/WCCC-899-50. Accessed 31 May 2020

Yue W, Changhong S, Wei Y (2010) Study of acquisition streetlights background signal by multi-sensor array. In: ICCAS 2010. IEEE, pp 1000-1003

Zanella A, Bui N, Castellani A, Vangelista L, Zorzi M (2014) Internet of Things for Smart Cities . Int Things J IEEE 1:22-32. https:// doi.org/10.1109/JIOT.2014.2306328

Zhihong Y, Yingzhao Y, Yu Y, Yufeng P, Xiaobo W, Wenji L (2011) Study and application on the architecture and key technologies for IOT. In: 2011 international conference on multimedia technology, 26-28 July 2011, pp 747-751. https://doi.org/10.1109/ ICMT.2011.6002149

Publisher's Note Springer Nature remains neutral with regard to jurisdictional claims in published maps and institutional affiliations. 